Document downloaded from:

http://hdl.handle.net/10251/36360

This paper must be cited as:

Ortigosa Araque, N.; Morillas Gómez, S.; Peris Fajarnes, G.; Dunai Dunai, L. (2012). Fuzzy Free Path Detection based on Dense Disparity Maps obtained from Stereo Cameras. International Journal of Uncertainty, Fuzziness and Knowledge-Based Systems. 20(2):245259. doi:10.1142/S0218488512500122.

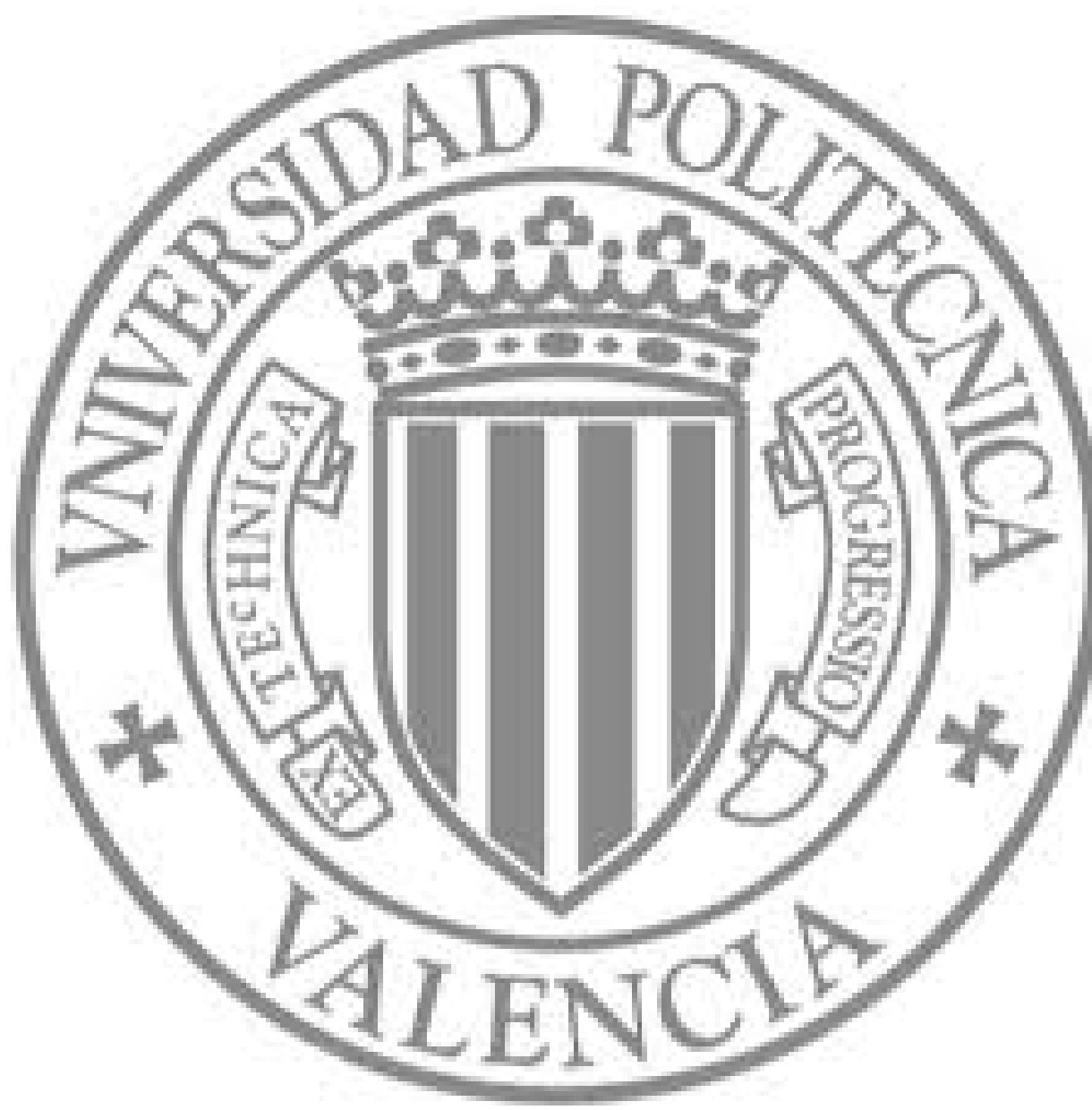

The final publication is available at

http://dx.doi.org/10.1142/S0218488512500122

Copyright World Scientific Publishing 
$\begin{array}{lllll}\text { January } & 9 & 2012 & 14: 25 & \text { WSPC/INSTRUCTION }\end{array}$ FreePathDetectionÚsingFuzzyLogic

International Journal of Uncertainty, Fuzziness and Knowledge-Based Systems

(C) World Scientific Publishing Company

\title{
FUZZY FREE PATH DETECTION BASED ON DENSE DISPARITY MAPS OBTAINED FROM STEREO CAMERAS
}

\author{
NURIA ORTIGOSA \\ Centro de Investigación en Tecnologías Gráficas \\ Universidad Politécnica de Valencia \\ Camino de Vera s/n, 46022 Valencia, Spain \\ nuorar@upvnet.upv.es \\ SAMUEL MORILLAS \\ Instituto Universitario de Matemática Pura y Aplicada \\ Universidad Politécnica de Valencia \\ Camino de Vera s/n, 46022 Valencia, Spain \\ smorillas@mat.upv.es \\ GUILLERMO PERIS-FAJARNÉS, LARISA DUNAI \\ Centro de Investigación en Tecnologías Gráficas \\ Universidad Politécnica de Valencia \\ Camino de Vera $s / n$, 46022 Valencia, Spain \\ Received (received date) \\ Revised (revised date)
}

\begin{abstract}
In this paper we propose a fuzzy method to detect free paths in real-time using digital stereo images. It is based on looking for linear variations of depth in disparity maps, which are obtained by processing a pair of rectified images from two stereo cameras. By applying least-squares fitting over groups of disparity maps columns to a linear model, free paths are detected by giving a certainty using a fuzzy rule. Experimental results on real outdoor images are also presented.
\end{abstract}

Keywords: Fuzzy logic, Computer Vision, Stereo Vision, Assisted Navigation.

\section{Introduction}

Obstacle avoidance is an essential task for intelligent automotive and robotic applications. Regarding this field, there are several works to aid automotive navigation without collisions. For example, Grosso and Tistarelli analyze the time-to-impact to objects in the scene to detect free space ${ }^{1}$, Kubota et al. find the optimum roadobstacle boundary to facilitate the auto-navigation of a vehicle ${ }^{2}$, and Franke and Joos apply distance dependent thresholds in disparity maps to analyze urban traffic ${ }^{3}$. Meanwhile, Nguyen et al. ${ }^{4}$, Badino et al. ${ }^{5}$ and Wedel et al. ${ }^{6}$ obtain information from a stereo vision system using the Sum of Absolute Differences between images, processing occupancy grids, and parametric B-splines, respectively. 
During last years, fuzzy logic has become a very useful tool to decision-making and reasoning. Specifically, for image processing, several works have used it with different objectives ${ }^{7}$. For instance, to represent spatial relations between different components in a scene by using membership functions ${ }^{8}$, to develop a method for moving objects segmentation and tracking ${ }^{9}$, or to efficiently suppress Gaussian and impulse noise from images by using fuzzy peer groups ${ }^{10}$. Many authors have also used fuzzy logic in order to address the problem of obstacle avoidance. For example, to build maps for robot navigation ${ }^{11}$, to analyze motion problems in a mobile robot equipped with ultrasound sensors ${ }^{12}$, to calculate the distance to an object to determine the next movement of a robot ${ }^{13}$, to avoid dynamic obstacles and limit free-space in predefined paths 141516 or to generate a neural network to detect obstacles onboard a vehicle ${ }^{17}$.

In this sense, it is important to remark that detecting free paths instead of detecting the obstacles of the scene represents an alternative point of view in navigation assistance. Indeed, there are a great number of references for obstacle detection and not so many for free space detection. Free path detection offers an advantage over the obstacle detection: free pathways are easier to detect by looking at the pattern they follow in a disparity map obtained by processing two stereo images. In disparity maps, disparity values decrease linearly from the bottom of the image to the top in obstacle-free areas, so free paths can be detected faster with less computational cost looking for pixels that match this pattern. Thus, we propose to detect this linear behaviour in order to detect free paths.

This paper presents an algorithm for the detection of free paths in real-time, to improve a previous crisp method ${ }^{18}$ which is integrated in the Cognitive Aid System for Blind and Partially Sighted People (CASBliP) project ${ }^{19}$. The main aim of CASBliP is to develop a system capable of interpreting and managing real world information from different sources to support mobility assistance to any kind of visually impaired users. This way, it assists the users to navigate their way outdoors along pavements. The main difference between CASBliP Project and other references which also relay scene information to the blind user, as ${ }^{20}$, lies in the portability of the system. In CASBliP, the device works onboard the visually impaired person. Thus, stereo cameras are constantly moving and the scene often contains blurred and deformed objects. The motivation of this work is the necessity of detecting surrounding obstacle-free space in real-time to assist in navigation applications.

The paper is organized as follows. The system where the proposed algorithm is included and the method to obtain disparity maps are described in Section 2. Section 3 details the proposed method for free paths detection and Section 4 presents experimental results. Finally, conclusions are drawn in Section 5. 


\section{Disparity maps}

In order to obtain the stereo images, two Firewire CCD cameras providing 240x320 pixel images have been used. The intrinsic and extrinsic parameters of the two cameras are precomputed using a classic chart-based calibration technique ${ }^{21}$.

In recent years, there has been considerable interest in dense depth estimation 22 . We have experimented with several methods, including belief propagation ${ }^{23},{ }^{24}$, dynamic programming ${ }^{25}$, and sum of squared differences with iterative aggregation ${ }^{26}$. Finally, we have chosen the approach described in ${ }^{25}$ to estimate disparity maps from each pair of stereo images, since this method is based on dynamic programming and it provides a good trade-off between quality of results and computational efficiency (around 8-10 frames per second on a $1.75 \mathrm{GHz}$ laptop). In Figure 1 we show two examples of stereovision images and the corresponding disparity maps computed.

In ${ }^{25}$, disparity maps are represented as $N \times M$ gray scale images, where darker areas are associated with further regions of the scene, as we can observe in Equation 1 , where $f$ is the focal length, $B$ is the distance between stereo-cameras and $d$ is the disparity between stereo-images ${ }^{27}$.

$$
Z=\frac{f B}{d}
$$

Most of the reported works in obstacle-free areas detection, use images captured from a stereo-vision system, since the use of stereo cameras allows the calculation of disparities for each pixel in every frame, which is a key feature to perform an accurate detection. The disparity of an image pixel $D(i, j)$ (where $(i, j)$ denote the pixel position in the image) refers the location difference between the pixel in the
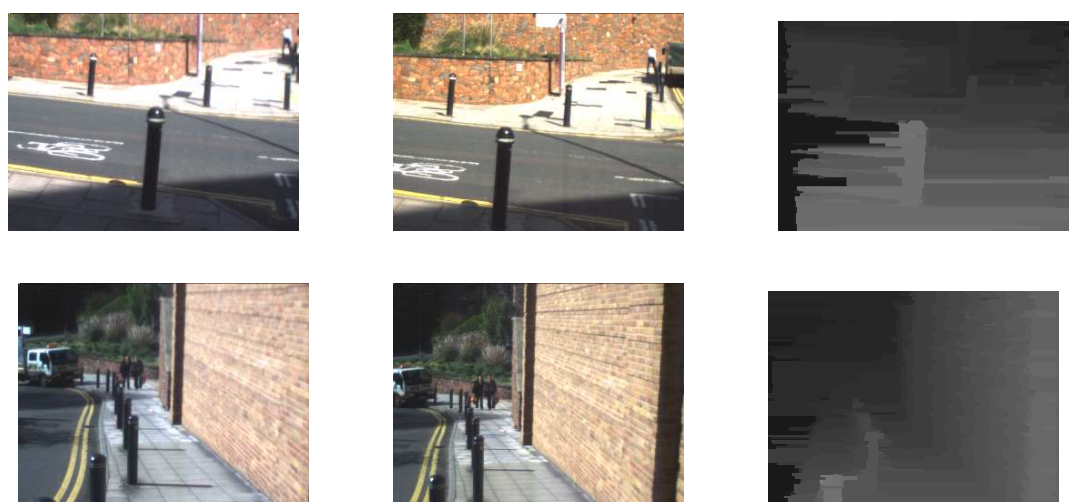

Fig. 1. Examples of stereo images and disparity maps. Left and right images are shown in first and second columns respectively and third column shows the corresponding disparity maps computed using the reference chosen. 
left image and the corresponding pixel in the right image after both images have been rectified. Clearly, the lower the disparity for a pixel is, the higher the distance up to the point represented by this pixel.

We propose to determine obstacle-free paths by processing only the $25 \%$ last rows of the disparity maps (from row 1 to row $N / 4$, using a coordinate system positive upwards), since these rows represent the region that the user will first walk through. This would also help the system to work in real-time and reduce the computational cost of the algorithm.

\section{Proposed Free Path Detection}

In this section, the proposed detection method is presented. It is based on the fuzzification of the detection method presented in ${ }^{18}$. The crisp detection algorithm is based on the fact that disparity map grey levels in obstacle-free areas decrease slightly and linearly from the bottom of the map to the top. In addition, obstacles for which depth is approximately constant are represented by flat zones. Two examples in Figure 2 illustrate this behaviour.

In the first example of Figure 2, for the free path of the column marked in the image and the respective disparity map (rows from 1 to 120), the graphic of the
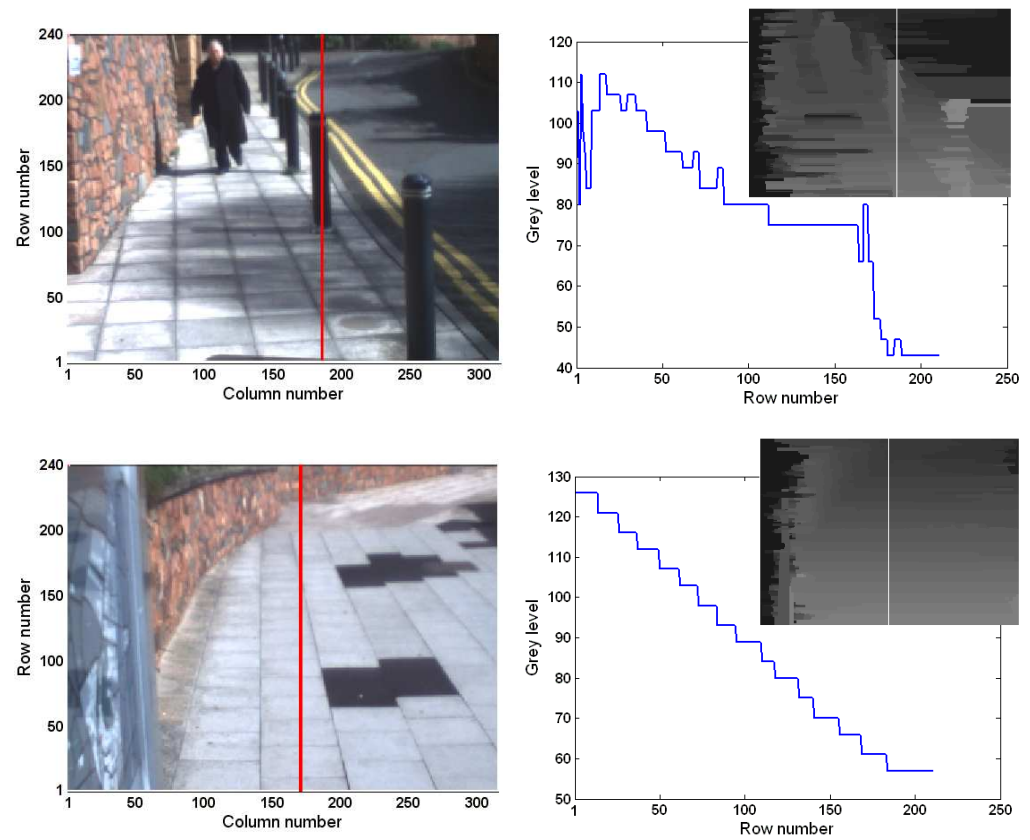

Fig. 2. Examples of free paths and obstacles behaviour in disparity maps. First column show left images from the stereo vision system. Second column show disparity maps associated and the representation of grey levels of the marked columns in the images. 
second column shows that grey levels of the disparity map decrease linearly from the bottom of the image (row 1 ) to the top of the disparity map. The pole (rows 120 to 170 ) is represented as a constant grey level. Obstacle-free area after the pole (rows from 170 on) has grey level variations, but not following a totally linear model, since in this case the disparity map presents errors when calculating the matching areas, that can be interpreted as noise.

In the second example of Figure 2, the marked columns in the image and the disparity map indicate a free path. In the associated representation of the grey levels, it can be observed that they decrease slightly and linearly from row 1 on (from the bottom of the image to the top). Thus, the presented method is based on the fact that disparity map column values of free paths can be adjusted to a linear model by using least-squares fitting as we will see in the following.

So, given a left image $I_{L}$ and a right image $I_{R}$ from a stereo-vision system, the disparity computed ${ }^{25}$ for pixel in (row, column) location $(i, j)$ is denoted by $D(i, j)$. In order to save computation time and also to reduce the influence of present noise due to wrong matchings, we process the disparity maps by averaging groups of $G$ pixel columns. Thus, we obtain the averaged column $\widetilde{D}(i, k)$ from the values of columns

$$
\begin{gathered}
D(i, j), j=G k-(G-1), G k-(G-2), G k-(G-3), \ldots, G k \\
\widetilde{D}(i, k)=\frac{D(i, G k-(G-1))+D(i, G k-(G-2))+\ldots+D(i, G k-1)+D(i, G k)}{G}
\end{gathered}
$$

where $i=N, N-1, \ldots, N-N / 4$ and $k=1,2, \ldots, M / G$. Then, a least-squares fitting over $\widetilde{D}(i, k)$ is done to find the best linear fitting to adjust the averaged column points. This model, which provides an estimate for $\widetilde{D}(i, k)$, is given by:

$$
\hat{D}(i, k)=a_{k} i+b_{k}
$$

where $i$ is the row number in the processed column of the disparity map, $\hat{D}(i, k)$ is the estimated disparity, $a_{k}$ is the gradient and $b_{k}$ is the y-intercept of the linear model. Regarding the obtained fitting, its coefficient of determination ${ }^{28}$ is defined as

$$
I(k)=\frac{\sum_{i=1}^{N / 4}(\hat{D}(i, k)-\bar{D})^{2}}{\sum_{i=1}^{N / 4}(\widetilde{D}(i, k)-\bar{D})^{2}},
$$

where $\bar{D}$ denotes the mean of the values in $\widetilde{D}(i, k) . I(k)$ measures the goodness of the fit for the group of columns $D(i, j), j=G k-(G-1), G k-(G-2), G k-$ $(G-3), \ldots, G k . I(k)$ may take values in $[0,1]$, where 0 means no correlation and 1 indicates a perfect correlation. 
According to above, ${ }^{18}$ determines as free paths only those groups of columns for which we obtain a good adjustment to the linear model. When processing an averaged column, the proposed detection method checks two different relations to perform the detection: it is checked both if the values of each coefficient of determination, $I(k)$, are large and if the gradients $a_{k}$ of the least-squares linear fitting vary in a smoothly way, in a range of negative values.

This reasoning is expressed in vague terms. Since large and smooth are linguistic terms, they can be represented by using fuzzy logic ${ }^{29}$. Particularly, the objective of this work is to develop the detection algorithm minimizing its computational cost, due to the hardware and real-time restrictions imposed. Thus, we propose to use the minimum number of linguistic variables and fuzzy rules to reduce the computational cost. As a result, the membership degree in the fuzzy set free-path is computed using the following fuzzy rule:

Fuzzy Rule 1: Determining the certainty of $\widetilde{D}(i, k)$ to be free path

IF "I $(k)$ is large" $A N D$ " $a_{k}$ is smooth"

$O R$

IF "I $(k)$ is large" $A N D$ " $a_{k}$ is very smooth"

OR

IF "I $(k)$ is medium" $A N D$ " $a_{k}$ is smooth"

THEN "the certainty of $\widetilde{D}(i, k)$ to be free path is high".

A group of columns is considered as free-path if 1)it fits the linear least-squares fitting where the disparities decrease along the columns with a smooth negative gradient, 2)if the coefficient of determination of the obtained fitting is large, although its gradient is very smooth (but negative), or 3)if the coefficient of determination is medium (not a high goodness of fit of the lineal model) but the gradient is negative and varies in a smoothly way.

In order to compute the membership degree in the fuzzy set large we need to know the desired behaviour, i.e., if the coefficient of determination is large enough, we want to have a large membership degree, but after a certain point, we want to decrease the membership degree according to the smaller values of the coefficient of determination. Therefore, we have chosen a trapezoidal membership function over other possible functions, due to its simplicity. This function is defined as follows:

$$
\mu^{I}(x)= \begin{cases}\frac{1}{\gamma_{1}} x & \text { if } 0 \leq x \leq \gamma_{1} \\ 1 & \text { if } \gamma_{1}<x \leq \gamma_{2} \\ \frac{-1}{1-\gamma_{2}} x+\frac{1}{1-\gamma_{2}} & \text { if } \gamma_{2}<x \leq 1\end{cases}
$$

where the values of the parameters $\gamma_{1}$ and $\gamma_{2}$ will be discussed in Section 4.1.

Regarding the membership degree on the fuzzy set smooth applied to the gradient, we want to have a large membership value for a range of negative values and, if it is out of that range, we want to decrease the membership slowly. Again, for simplicity, we have chosen a trapezoidal membership function, which is defined as 
follows:

$$
\mu^{a}(x)= \begin{cases}0 & \text { if } x \leq \gamma_{3} \\ \frac{1}{\gamma_{4}-\gamma_{3}} x-\frac{\gamma_{3}}{\gamma_{4}-\gamma_{3}} & \text { if } \gamma_{3}<x \leq \gamma_{4} \\ 1 & \text { if } \gamma_{4}<x \leq \gamma_{5} \\ \frac{1}{\gamma_{5}} x & \text { if } \gamma_{5}<x \leq 0 \\ 0 & \text { if } x>0\end{cases}
$$

where the values of the parameters $\gamma_{3}, \gamma_{4}$ and $\gamma_{5}$ are negative, and will be discussed in Section 4.1.

The fuzzy sets medium and very smooth can also be modelled by using the functions defined above, just varying the values of the parameters $\gamma_{1}, \gamma_{2}$ and $\gamma_{3}, \gamma_{4}, \gamma_{5}$ from Equations 5 and 6, respectively. These values will be discussed in Section 4.1.

Figures 3(a) and 3(b) show the membership functions for the coefficient of determination and the gradient, respectively.

The fuzzy rule 1 contains three conjunctions and two disjunctions. In fuzzy logic, t-norms and co-norms are used to represent conjunctions and disjunctions, respectively ${ }^{30},{ }^{31},{ }^{32}$. The obstacle-free degree of $\widetilde{D}(i, k)$ is computed using the product triangular norm to represent the fuzzy $A N D$ (conjunction) operator and the probabilistic sum co-norm to represent the fuzzy $O R$ (disjunction) operator. Since we aim at computing a fuzzy obstacle-free degree, no defuzzycation is needed. Algorithm 1 details the proposed fuzzy detection algorithm.

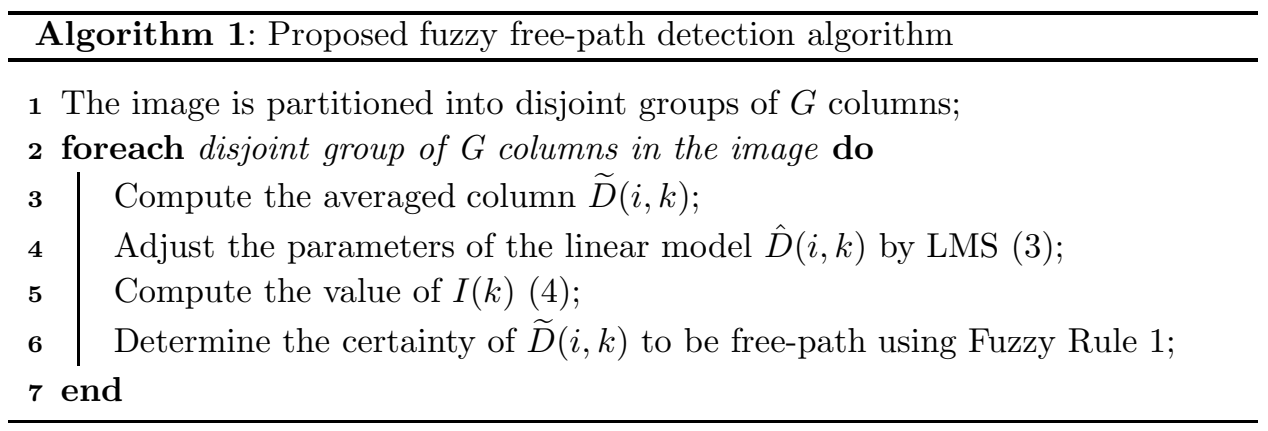

\section{Experimental results}

In order to objectively measure the detection algorithm performance, it was necessary to manually prepare some groundtruth images, in which each pixel was marked as free-space or occupied by some object. This way, the detected areas can be compared with the groundtruth images pixel by pixel in order to obtain objective assessments of the detection performed. We use the mean square error (MSE) to measure the goodness of the detection method proposed. 
$\begin{array}{lllll}\text { January } & 92 & 2012 & 14: 25 & \text { WSPC/INSTRUCTION }\end{array}$

\section{FreePathDetectionÚsingFuzzyLogic}

8 N. Ortigosa, S. Morillas, G. Peris-Fajarnés, L. Dunai

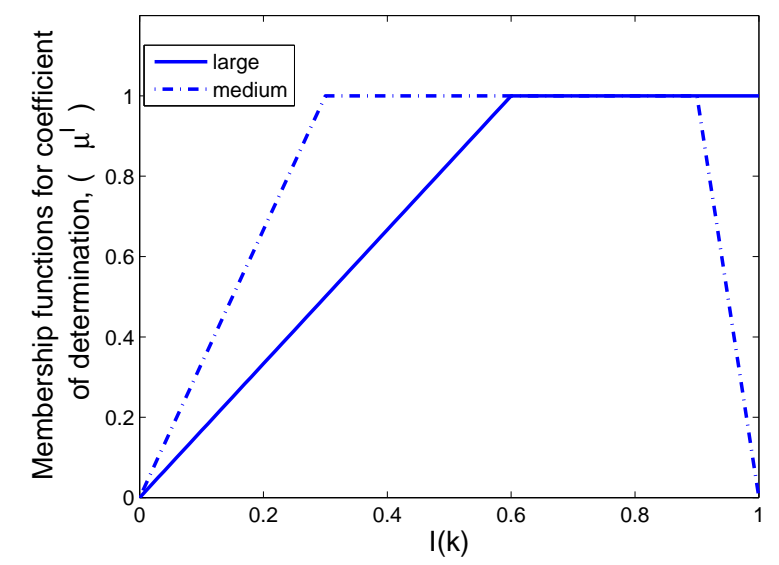

(a)

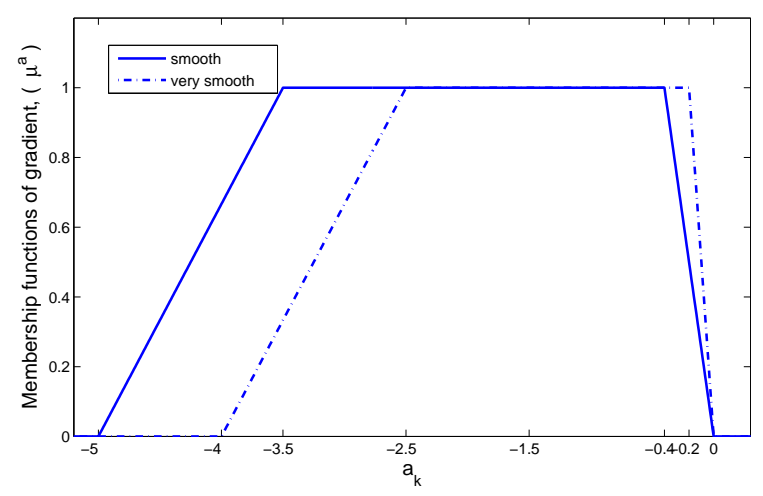

(b)

Fig. 3. Membership functions: (a) Membership functions large and medium for the coefficient of determination $I(k)$, (b) Membership functions smooth and very smooth for the gradient $a_{k}$.

\subsection{Parameters Adjustment}

Determining an appropriate parameter adjustment is a very important task that has to be carried out in order to obtain successful detection results. We have taken a training image set of 35 real outdoor images and we have manually prepared their corresponding groundtruths. This training set includes the most common scenarios that a person can run into outdoors, such as cars, poles, pedestrians, walls, obstaclefree areas, etc. These images have been taken in different illumination conditions, since the quality of disparity maps used in the algorithm detection depends on the illumination conditions of the scene and, as a result, as we base our detection method in the analysis of variations in disparity maps, this affects our algorithm. In everyday outdoor scenarios, non-ideally illuminated situations often occur. Such conditions lead the stereo correspondence algorithms to many false-matchings, since 
this implies that it is more difficult to find the disparity between the left and right images from the stereo-vision system ${ }^{33}$. Thus, it is necessary to include different illumination situations in the training image set.

As it is desirable to have settings that allow the method to perform well for a variety of different outdoor scenarios, we have found the suboptimal values for the parameters that define the membership functions, in order to minimize the mean square error for the whole training set.

We have experimentally found that the parameters should be fixed as follows:

- Large membership function for $I(k): \gamma_{1_{L}}=0.6, \gamma_{2_{L}}=1$

- Medium membership function for $I(k): \gamma_{1_{M}}=0.3, \gamma_{2_{M}}=0.9$

- Smooth membership function for $a_{k}: \gamma_{3_{S}}=-5, \gamma_{4_{S}}=-3.5, \gamma_{5_{S}}=-0.4$

- Very smooth membership function for $a_{k}: \gamma_{3_{V S}}=-4, \gamma_{4_{V S}}=-2.5, \gamma_{5_{V S}}=$ $-0.2$

\subsection{Algorithm performance}

In this section we assess the performance of the free-path detection method, using the previous settings. Second row of Figure 4 shows some test images and Table 1 shows the performances for the testing image set compared with the crisp reference 18 (Figure 4, first row). These figures confirm that the algorithm works and detects free-paths properly, with values of MSE smaller than 0.12 in average for the testing image set (which is different from the training image set used for the parameters adjustment).

However, disparity maps present errors that can be considered as noise, due to wrong matchings (Figure 5). In order to provide robustness against noise, we propose to remove flat black zones (which correspond to occlusions from the matching process) and remove pixels whose gradient along the column varies indicating abrupt depth changes, too. We consider that we have abrupt depth changes when disparity changes in more than 10-15 pixels from one row to another which is next to it (by using Equation 1 this means about 5 meters of depth change in our system). Thus, we remove from our study disparity map pixels that hamper to have linear decreasing behaviour in obstacle-free areas, before performing the least-squares fitting. We can observe that the Mean Square Error decreases $46.22 \%$ and $18 \%$ for the crisp and fuzzy detection, respectively, after denoising the disparity maps. 
\begin{tabular}{|lllll} 
January & 9 & 2012 & $14: 25$ & WSPC/INSTRUCTION
\end{tabular} FreePathDetectionÚsingFuzzyLogic
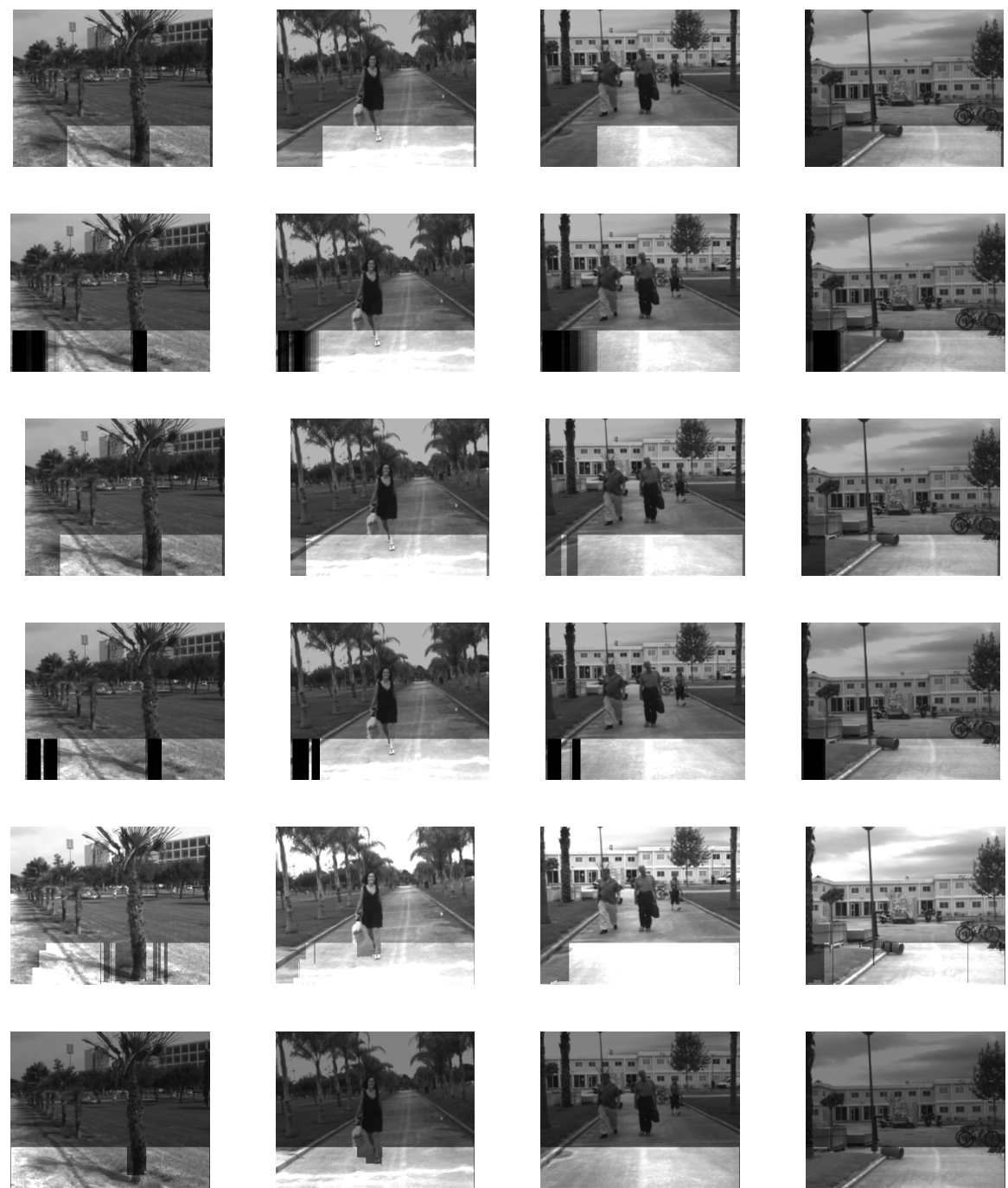
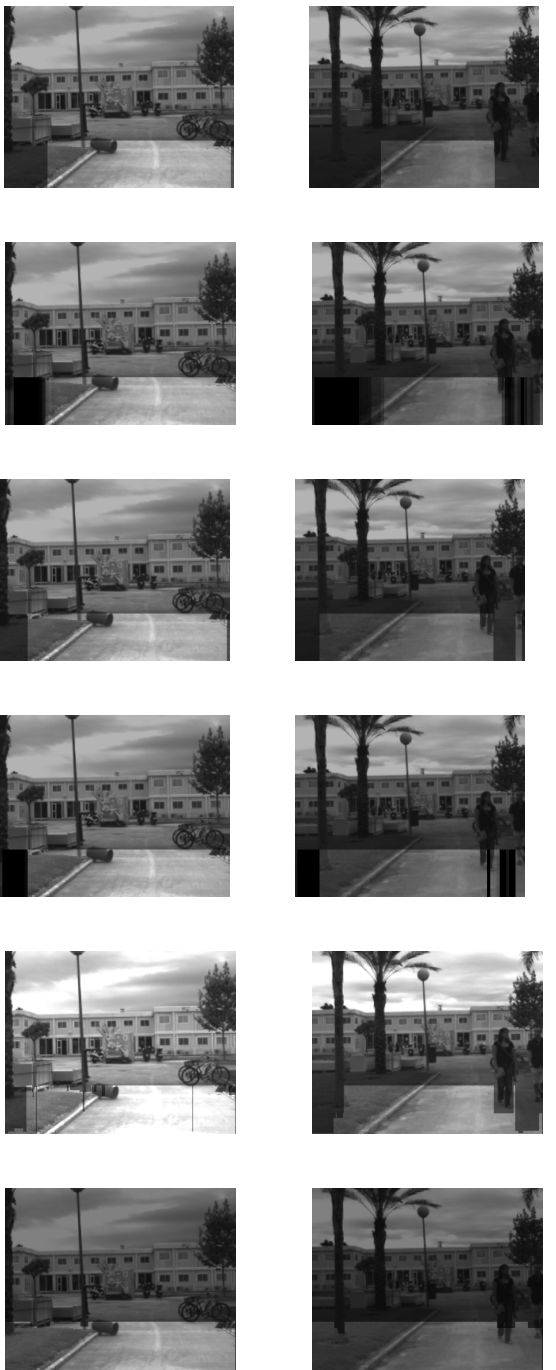

Fig. 4. Examples of testing images set. Brighter areas show higher certainties of the detected areas to be free paths. First row shows results of the crisp method proposed in ${ }^{18}$, second row show the performance of the proposed method, third and fourth rows show crisp and fuzzy results with improved (reduced noise) disparity maps, and fifth and sixth rows show ${ }^{9}$ and ${ }^{6}$ results, respectively. 
Table 1. Mean square error of the fuzzy detection method for the testing image set, compared with the crisp reference ${ }^{18}$, using disparity maps with noise and denoised. Columns fifth and sixth show performances for two recent references.

\begin{tabular}{ccccccc}
\hline & Crisp & Fuzzy & Denoised crisp & Denoised fuzzy & 5 & 2 \\
\hline Average & 0.34 & 0.12 & 0.18 & 0.09 & 0.19 & 0.03 \\
Minimum & 0.22 & 0.08 & 0.10 & 0.05 & 0.14 & 0 \\
Maximum & 0.57 & 0.17 & 0.28 & 0.13 & 0.30 & 0.08 \\
\hline
\end{tabular}

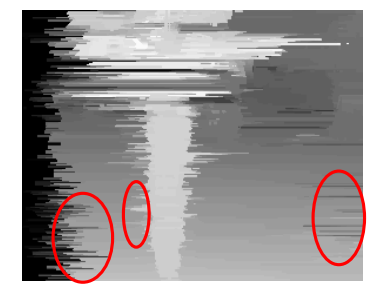

Fig. 5. Disparity map with noise. In red are marked the zones where we propose to reduce noise.

The performance of the different methods can be compared in Figure 4 and Table 1. Results show that the proposed method is able to outperform some of the most recent references, such as ${ }^{5}$ and ${ }^{18}$. The only method that performs better than the presented method in this paper is the proposed in ${ }^{2}$. However, it employs Disparity Space Images as initial matching cost in the algorithm to obtain the disparity maps and it is not completely comparable, since we use dense disparity maps. In any case, Kubota's method is not appropriated for the navigation aid system where the presented work is integrated, since it needs special hardware requirements (such as an FPGA) to provide results in real-time. In our case, since the system works onboard the visually impaired person, it is necessary to reduce its weight as much as possible. So, minimal hardware requirements and computational cost to facilitate running in real-time in a small laptop are restrictions which are imposed to our method. This has also limited the use of a more complex fuzzy system that improves the performance, due to the increment of computational cost that it implies.

Moreover, the performance of the presented method highly depends on the accuracy of the disparity maps. The visual analysis of Figure 4 reveals that sometimes free paths are classified as obstacles. Usually, these areas correspond to borders of the image, untextured areas (such as asphalt) or complex situations where disparity cannot be accurately computed, which could lead to low performance. Also, small objects placed quite far from the user (for example, the foot of the girl in the sec- 
ond column of Figure 4) can not be classified as an obstacle. This is caused by errors in the associate disparity values. However, as the presented algorithm works in real-time at around 8 frames per second, in the following frames these small objects whose wrong obtained disparity can induce errors will become bigger objects, making easier to obtain the correct associate disparities. Thus, the algorithm will provide better detection results for small objects as long as the user of the system is nearer to the objects. Using Equation 1 we can estimate that objects bigger than $7 \mathrm{~cm}$ at distances nearer that $2.54 \mathrm{~m}$ are properly detected, which is enough to assist blind users outdoors at a comfortable walking speed ${ }^{34}$.

Finally, Figure 6 shows examples of the algorithm's output. Brighter areas indicate that their certainty to be a free-path is higher than the darker ones. Figures confirm that the algorithm works well and detects free paths, reducing the Mean Square Error a $47.58 \%$ and a $65.62 \%$ compared to the crisp detection of the algorithm, with or without noise reduction in disparity maps, respectively. It always provides the wider obstacle-free area to walk through, so the method performs appropriately for the purpose of the project in which it has to be integrated, in order to avoid collisions of the end user. Moreover, the fuzzy algorithm is suitable for real-time working, which is essential to be integrated as a support to navigate for blind and visually impaired people. Its implementation in C programming language is able to process 8-10 frames per second in a $1.73 \mathrm{GHz}$ and 1 GB RAM laptop, which is enough to assist visually impaired users to navigate outdoors without collisions.

\section{Conclusions and future work}

In this paper a new fuzzy method for free path detection has been proposed. It is based on an analysis of disparity maps obtained by processing a pair of images from two stereo cameras. The method is based on a fuzzy logic system that assigns a certainty to each pixel of being an obstacle-free area depending on the fitting to a linear model. We use the adjustment of the column grey levels of the disparity maps by least-squares. Experimental results on real outdoor images show that the method provides promising results. The algorithm offers real-time performance. Future work will focus on improving performance, in order to realize a continuous detection by including temporal coherence. 
$\begin{array}{lllll}\text { January } & 9 & 2012 & 14: 25 & \text { WSPC/INSTRUCTION }\end{array}$ FreePathDetectionÚsingFuzzyLogic
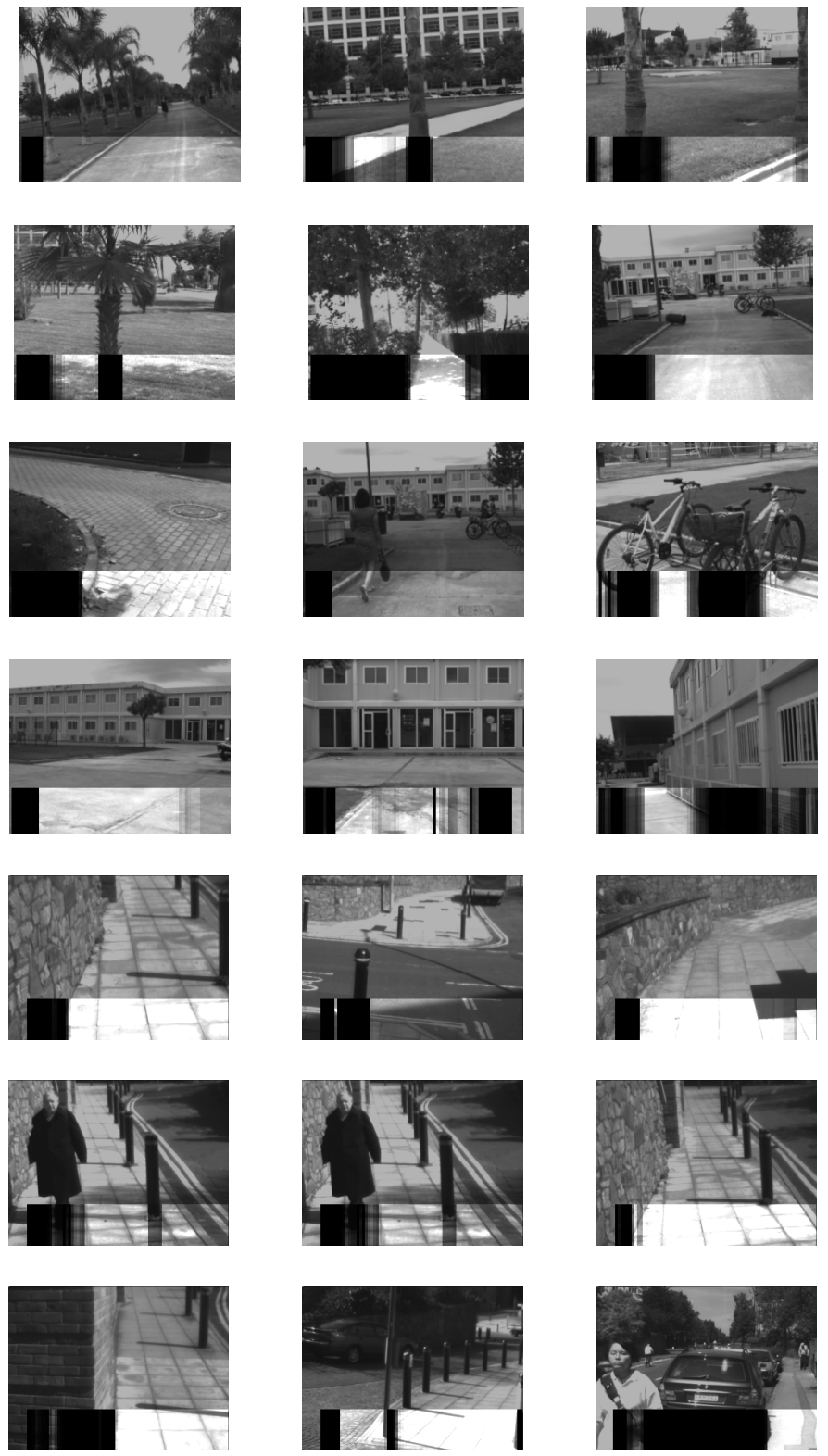

Fig. 6. Processed testing images set. Brighter areas show detected free-paths. Darker areas indicate that they have smaller certainties to be an obstacle-free path. 


\section{References}

1. E. Grosso and M. Tistarelli. Active/dynamic stereo vision. IEEE Transactions on Pattern Analysis and Machine Intelligence, 17(9):868-879, September 1995.

2. S. Kubota, T. Nakano, and Y. Okamoto. A global optimization for real-time on-board stereo obstacle detection systems. In IEEE. IEEE Intelligent Vehicles Symposium, June 2007.

3. U. Franke and A. Joos. Real-time stereo vision for urban traffic scene understanding. In IEEE. IEEE Intelligent Vehicles Symposium, October 2000.

4. T. H. Nguyen, J.S. Nguyen, D.M. Pham, and H.T. Nguyen. Real-time obstacle detection for an autonomous wheelchair using stereoscopic cameras. Conf Proc IEEE Eng. Med. Biol. Soc., 2007(1):4775-4778, 2007.

5. H. Badino, R. Mester, T. Vaudrey, and U. Franke. Stereo-based free space computation in complex traffic scenarios. pages 189-192. IEEE Southwest Symposium on Image Analysis \& Interpretation, 2008.

6. A. Wedel, H. Badino, C. Rabe, H. Loose, U. Franke, and D. Cremers. B-spline modeling of road surfaces with an application to free-space estimation. IEEE Transactions on Intelligent Transportation Systems, 10(4):572-583, December 2009.

7. I. Bloch. Fuzzy spatial relationships for image processing and interpretation: a review. Image and Vision Computing, 23(2):89-110, February 2005.

8. J.M. Keller and X. Wang. A fuzzy rule-based approach to scene description involving spatial relationships. Computer Vision and Image Understanding, 80(1):21-41, October 2000.

9. J. Moreno-Garcia, L. Rodriguez-Benitez, A. Fernandez-Caballero, and M.T. Lopez. Video sequence motion tracking by fuzzification techniques. Applied Soft Computing, 10(1):318-331, January 2010.

10. S. Morillas, V. Gregori, and A. Hervas. Fuzzy peer groups for reducing mixed gaussianimpulse noise from color images. IEEE Transactions on Image Processing, 18(7):14521466, July 2009.

11. M. Poloni, G. Ulivi, and M. Vendittelli. Fuzzy-logic and autonomous vehicles: Experiments in ultrasonic vision. Fuzzy Sets and Systems, 69(1):15-27, January 1995.

12. J.M. Alonso, L. Magdalena, S. Guillaume, M.A. Sotelo, L.M. Bergasa, M. Ocana, and R. Flores. Knowledge-based intelligent diagnosis of ground robot collision with non detectable obstacles. Journal of Intelligent and Robotic Systems, 48(4):539-566, April 2007.

13. A. Menon, R. Akmeliawati, and S. Demidenko. Towards a simple mobile robot with obstacle avoidance and target seeking capabilities using fuzzy logic. In Proceedings, volume 1-5, pages 1003-1008. IEEE Instrumentation and Measurement Technology Conference, 2008.

14. K. Kim, J.J. Park, M.S. Yang, O.S. Kwon, and E.H. Lee. Fuzzy logic-based path planning for a mobile robot. pages 343-350. 4th International FLINS Conference on Intelligent Techniques and Soft Computing in Nuclear Science and Engineering, August 2000 .

15. G. Kumar and V.P. ViJayan. A multi-agent optimal path planning approach to robotics environment. In Proceedings, pages 400-404. International Conference on Computational Intelligence and Multimedia Applications, 2007.

16. L. McFetridge and M.Y. Ibrahim. A new methodology of mobile robot navigation: The agoraphilic algorithm. Robotics and Computer-Integrated Manufacturing, 25(3):545551, June 2009.

17. H.J. Sun and J.Y. Yang. Obstacle detection for mobile vehicle using neural network and fuzzy logic. Neural Network and Distributed Processing, 4555(1):99-104, 2001. 
18. N. Ortigosa, S. Morillas, and G. Peris-Fajarnés. Obstacle-free pathway detection by means of depth maps. Journal of Intelligent and Robotic Systems, 63(1):115-129, July 2011.

19. http://www.casblip.com.

20. P.D. Picton and M.D. Capp. Relaying scene information to the blind via sound using cartoon depth maps. Image and Vision Computing, 26(4):570-577, April 2008.

21. Z.Y. Zhang. A flexible new technique for camera calibration. IEEE Transactions on Pattern Analysis and Machine Intelligence, 22(11):1330-1334, November 2000.

22. D. Scharstein and R. Szeliski. A taxonomy and evaluation of dense two-frame stereo correspondence algorithms. International Journal of Computer Vision, 47(1/2/3):742, April-June 2002.

23. P.F. Felzenszwalb and D.P. Huttenlocher. Efficient belief propagation for early vision. International Journal of Computer Vision, 70(1):41-54, October 2006.

24. Q. Yang, L. Wang, R. Yang, H. Stewénius, and D. Nistér. Stereo matching with colorweighted correlation, hierarchical belief propagation, and occlusion handling. IEEE Transactions on Pattern Analysis and Machine Intelligence, 31(3):492-504, March 2009.

25. S. Birchfield and C. Tomasi. Depth discontinuities by pixel-to-pixel stereo. International Journal of Computer Vision, 17(3):269-293, December 1999.

26. C. Lawrence Zitnick and S. Bing Kang. Stereo for image-based rendering using image over-segmentation. International Journal of Computer Vision, 75(1):49-65, October 2007.

27. R. I. Hartley and A. Zisserman. Multiple View Geometry in Computer Vision. Cambridge University Press, ISBN: 0521540518, second edition, 2004.

28. M.R. Spiegel and L.J. Stepthens. Statistics. Mc Graw Hill, fourth edition, 2008.

29. E.E. Kerre. Fuzzy Sets and Approximate Reasoning. Xian Jiaotong Univ. Press, 1998.

30. D. Dubois and H. Prade. Fuzzy Sets and Systems: Theory and Applications. Academic Press, New York, 1980.

31. C.C. Lee. Fuzzy logic in control systems: Fuzzy logic controller-parts 1 and 2. IEEE Transactions on Systems, Man and Cybernetics, 20(2):404-435, March-April 1990.

32. J.C. Fodor. A new look at fuzzy-connectives. Fuzzy Sets and Systems, 57(2):141-148, July 1993.

33. L. Nalpantidis and A. Gasteratos. Stereo vision for robotic applications in the presence of non-ideal lightning conditions. Image and Vision Computing, 28(6):940-951, June 2010 .

34. Richard W. Bohannon. Comfortable and maximum walking speed of adults aged 2079 years: reference values and determinants. Age and Ageing, 26(1):15-19, January 1997. 